Conference Paper

\title{
Improvement Research PLC Automatic Control System Based on Small and Medium Logistics Classification
}

\author{
Zhengzheng Cong, Cong Li, Yifeng Shao, Zhize Zhou, Chunmeng Liang \\ Department of Mechanical Engineering, Guilin University of Aerospace Technology, Guilin, China
}

Email address:

Congzz@guat.edu.cn (Zhengzheng Cong), licong@guat.edu.cn (Cong Li), 369468271@qq.com (Yifeng Shao), 2507663523@qq.com (Zhize Zhou),7608782202@qq.com (Chunmeng Liang)

\section{To cite this article:}

Zhengzheng Cong, Cong Li, Yifeng Shao, Zhize Zhou, Chunmeng Liang. Improvement Research PLC Automatic Control System Based on Small and Medium Logistics Classification. Automation, Control and Intelligent Systems. Vol. 3, No. 6, 2015, pp. 124-127.

doi: 10.11648/j.acis.20150306.15

\begin{abstract}
PLC automatic control classification system is based on commitment to increase express courier company and distribution center for the delivery of processing power, making it more efficient for the shipment have free processing capabilities. Based on the scientific method, prior to the courier company handling capacity estimates, and the company in shipment processing equipment capital investment to enhance the product in the industry's competitiveness. It is used of the existing two-dimensional code scanning recognition technology. Through the automatic identification of microcontroller programming combined ratio for the sensor to achieve classification. It works to reduce logistics costs in the classification of goods while improving the efficiency and accuracy of their goods classification.
\end{abstract}

Keywords: Intelligent Classification, High Precision, Continuous Work

\section{Study Background}

Based on all of today's domestic shipments by province and country are required for centralized delivery sorting process conditions, resulting in a shipment of more complex and requires a large amount and classification and timely comparison. Due to the rapid development of the courier company, leading to the sorting process is now also fully express stuck in backward manual processing stages. Thus, it affects the efficiency of logistics and accuracy under these conditions the corresponding article classifier ready. Swift classification treasure is mainly used in the classifying of goods, such as express delivery companies to provinces or countries classified items classified as other fields. Mainly used to automate classification of goods, to reduce costs and improve the accuracy and efficiency of classification of goods. Using infrared scanning technology to items brought two-dimensional code identification, and the two-dimensional code information passed SCM console. SCM through incoming digital into analog control amount, and then by the microcontroller output control of the state sensor. Control then transferring articles through the system. After collected the respective collector items, the collector sensor status changes, the items falls into the corresponding collector. Thus, it makes goods classification.

In order to achieve efficient and accurate classification of goods as the goal. Swift is expected to classification efficiency classification Per 1200 per hour, while the artificial classification of up to 160 bags per hour and cannot continue to work more than eight hours, the specifications for the mass of less than $25 \mathrm{~kg}$, volume in the $80 * 80 * 100$ of items.

\section{The Research Objectives}

This work is the key technology of the microcontroller can be programmed in advance, and then use the MCU receives sensor status signal and infrared scanner to approach address information, after the microcontroller logic processing, and then send control commands to control the motor to rotate and turn the dial to collect cells from the MCU The opening and closing. Applied to the microcontroller, angle sensors, two-dimensional code scanner, infrared sensors and other equipment. Relatively conventional automatic sorting system, which works great collection device using rotary machines reduce the space occupied, with a high degree of intelligence, 
an area of small, low one-time cost, classification accuracy and efficiency advantages.

\section{Assessment Indicators}

It works with the current emerging two-dimensional code technology and MCU technology and sensor technology the perfect combination to form a complete set of goods classification system. PLC automatic control system design for improved innovation goods classification based collector, produced specifically for the goods classification to achieve automatic classification system design..The basic working principle is as follows: The microcontroller programming, scanner input address information items, then SCM issued a directive, the article collector on behalf of photo sensors that address open, when the article passes this time sensitive sensor is blocked. The transport board separate items falling from the transport board, falling into the article corresponding address slot, and then close the transport board, run-down, in the end portion, the process is repeated again.

\section{Based on PLC Automatic Control System}

China have also appeared suitable for small and medium sorter express courier company. But it's only sorting less than $5 \mathrm{~kg}$ of shipment, which for a little bulky courier cannot handle. However, companies developed this product also did not enter mass production stage. Current situation of the courier company working in classified areas, found that most of the small and medium sized courier companies have not achieved classified intelligence resulting in high costs, low classification efficiency, accuracy cannot be guaranteed. The main reason for this phenomenon is the capital weak small and medium companies, we cannot afford large-scale sorting systems high time investment. But the courier classification developments must be intelligent. Therefore, it is suitable for small and medium enterprises to improve the characteristics of a high level of intelligence, high efficiency, high precision, low cost classification system. Bold design conceived PLC automatic control of classification system based on: continuous, a large number of classification courier, using automatic assembly line work, and not subject to time, weather and human limitations can reach more than 100 hours of continuous work, and the number per unit time classification pieces more can be reached 900-1200 per hour, manual classification efficiency above 5 times. It was unable to continue high-intensity artificial classification 8 hours.

\section{The Content of the Research Projects, Research Objectives, and Key Scientific Problems to Be Solved}

The main research work items classification, such as parcel delivery company to classify and other fields. Articles are intended to achieve automatic classification, in order to improve the situation. Use two-dimensional code scanning technology for goods carried by the two-dimensional code recognition, and the two-dimensional code before the contents of incoming console and the program will be compiled and implemented through the MCU conversion logic functions than the right, and then by the microcontroller output sensor control state, the sensor status changes within the collector, and ultimately for goods classification. Technical key point in the realization of the main items of data within two-dimensional code to achieve the objects of the efficient and accurate classification. Targets will be achieved one day (10 hours) Category 2000, specifications for the mass of less than $20 \mathrm{~kg}$, volume in the $80 * 80 * 100$ of items. Classification efficiency is expected 900-1200 per hour continuous operation for 100 hours and a maximum of 160 artificial classification per hour and cannot continue to work more than eight hours. Products are not suitable for classification slim, long, overweight and classification of fragile items. Technology is the key to this work is that the microcontroller can be programmed, and then use MCU receives status signals and infrared sensor scanner scanned address information, after the microcontroller logic processing, they are send control commands to control motor rotation and then dial the collection grid by the microcontroller.

Works by the transportation system, QR code scanning system, computer network of single-chip microcomputer processing system, sensor devices and chassis. Transportation system is the important part of the conveyor belt and transmission wheel and the radial transmission device to collect one cell, it is mainly used for early express transportation and later will express delivery to the designated collection bag. QR code scanning system is the main component of $\mathrm{QR}$ code scanning guns, is used to identify within its code address information and the information transmitted to the computer. Single chip computer network system for receiving and processing signal and sent to transfer control instruction to realize automatic classification effect. Scanning system will address information transmitted to MCU. It commands to control rotate to the corresponding Angle control the radial transmission device to collect one cell will be Courier to the corresponding collection bags.

\section{Research Programs to Be Undertaken and Feasibility Analysis}

Design concept of the automatic control of PLC-based classification system, which not only has continuous work long hours, a lot of free delivery, the use of automatic assembly line work and so on. Due to stick with a bar code or two-dimensional code scanning input, express categorization process basically intelligent. Based on design ideas, this work consists of the following components: products from the transport system, two-dimensional code scanning system, 
computer network microcontroller processing systems, sensor devices and a rack.

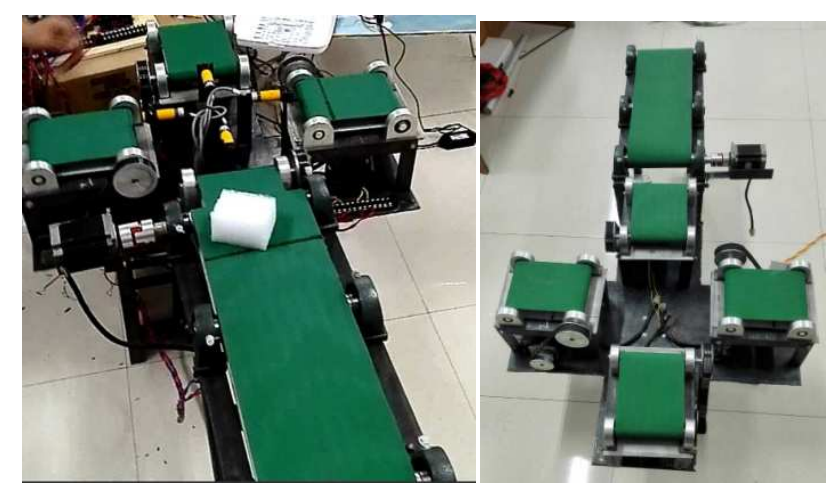

Figure 1. Accessories: computer console, motor, angle sensors, goods collection bag.

The main components of the transportation system and the radial transfer means collected within the cells, mainly for the early delivery of express transport and the latter will transfer to the designated collection bag. Dimensional code scanning system is a major component of the two-dimensional code scanner, for the two-dimensional code identifying the address information and transmits the information to the computer. Computer network microcontroller processing system for receiving, processing and transmission of the classification signal transmission control instructions to automate the classification results. Scanning system will address information is transmitted to the microcontroller. MCU sends commands to control turntable rotation radial transfer means collected within the cells control the collection bag when the courier transferred corresponding to the appropriate angle. The key technology is the process of operation, based on the input and output decency control PLC automatic control system programming and control accuracy as well as infrared scanning identification.

Simulation procedure is as follows: The workers will express a two-dimensional code face up on the first conveyor belt, when the courier run to the second conveyor at the infrared sensor, the signal is transmitted to a computer console, MCU sends control command control first conveyor stops 3S. In this case the courier is still on the second conveyor run, two-dimensional code and two-dimensional code scanner to scan information on the courier and the information is transmitted to the computer console. When the items are transported to the turntable collection grid, single-chip issue control instructions to the computer console before transmission based on a two-dimensional code address information, and precompiled SCM process, control the angle sensor to collect cells to be detected on the corresponding angle. If the turntable is rotated to the appropriate angle to collect cells such as 90 degrees, the computer console, this information is collected MCU issue control instructions to control the inner diameter corresponding to the transfer apparatus to collect cells will express came along and fall into the corresponding radial turntable collection bags, items at this time classification process is completed.
The production process:

First stage is according to the investigation team found that express company now as the low degree of intelligence, low efficiency, high accuracy cannot be guaranteed, artificial cost of these shortcomings. Accordingly we propose using the single chip microcomputer technology, Angle sensors, QR code scanner, infrared sensor technology, and computer network auxiliary processing information collection, each component of a feedback control instruction to complete intelligent classification.

Team in the second stage after a series of discussions on argument to start $3 \mathrm{~d}$ model of the product, and on this basis to modify the structure of the product, such as design team before spiral collection system and the automatic warehouse system because of the need to apply to the stack to implement more difficult, so later became now rotating disc collecting system in combination with Angle sensor technology and realize express simple and effective classification.

Third stage team began to design parts according to the established $3 \mathrm{~d}$ model of the machining drawing and purchase the required materials products and commissioning and correction.

Work instructions: Workers will have a parcel with QR code on the side as the first of the conveyor belt, when delivery to the second transmission with an infrared sensor, the signal is transmitted to the computer console, SCM control command control the first conveyor belt to stop $3 \mathrm{~s}$. At this point, the Courier is still in the second run on the conveyor belt, and QR code scanner scans the information on the delivery and will transfer the information to the computer console. When goods are transported to the turntable from SCM according to the information transmitted to a computer console QR code address before and compiled microcomputer program control instruction, beforehand control from the Angle of the sensors need to detect the corresponding point of view. When the wheel from rotation to the corresponding Angle such as 90 degrees, computer console to collect information about the single chip microcomputer control instruction control radial transmission device inside the corresponding collection. When express came along the rotary radial and fall into the corresponding collection bags, goods classification process is complete at this time.

\section{Conclusions}

Works applied to the microcontroller, angle sensors, two-dimensional code scanner, infrared sensors and other equipment. Relatively conventional automatic sorting system, which works great collection device using rotary machines reduce the space occupied. Combined with a microcontroller and sensor technology to automate the classification of a courier 3s, improves the classification efficiency. Technological innovation, new product so that the current two-dimensional code technology and MCU technology and sensor technology is the perfect combination to mix into a 
complete article classification system.

Thanks to the national college students' innovative entrepreneurial project funding support.

\section{References}

[1] CHENG Lei 1, XU Yu-xian 2, JIN Wei-xing 2 (1.Suzhou Institute of Industrial Technology, Suzhou Jiangsu 215104, China; 2. Suzhou Xinya Electronic Communication Co., Ltd., Suzhou Jiangsu 215132, China); Design of Connector Automatic Assembly Machine Based on the PLC [J]; Equipment Manufacturing Technology; 2012-07.

[2] WU Yi-zhong 1 GONG Yun-bo 1 ZHANG Guo-quan $\sim(2,3)$ (1.National CAD Support Software Engineering Research Center, Huazhong University of Science and Technology, Wuhan 430074, China; 2.Wuhan Polytechnic University, Wuhan 430023, China; 3. Wuhan Rentian Packaging Technology Co., Ltd., Wuhan 430205, China); Research on Key Technologies of Automatic Production Line of Industrial Explosive Secondary Packaging [A]; [C]; 2007.

[3] Gao De, Xie Hao (Engineering college, Jiamusi university, Jiamusi 154007, China); Develop and Research of Packing machinery MCAI [J]; PACKAGING ENGINEERING; 1999-04.
[4] Zhou Gao-fei; Research and Development of JQ 700 Type Box-bridge Erecting Machine [J]; Chongqing Architecture; 2008-05.

[5] L. Wang, Adaptive Fuzzy Systems and Control: Design and Stability Analysis, Prentice-Hall, Englewood Cliff, NJ, USA, 1994.

[6] R. M. Sanner and J.-J. E. Slotine, "Gaussian networks for direct adaptive control," IEEE Transactions on Neural Networks, vol. 3, no. 6, pp. 837-863, 1992.

[7] J. T. Spooner and K. M. Passino, "Stable adaptive control using fuzzy systems and neural networks," IEEE Transactions on Fuzzy Systems, vol. 4, no. 3, pp. 339-359, 1996.

[8] M. Liu, "Decentralized control of robot manipulators: nonlinear and adaptive approaches," IEEE Transactions on Automatic Control, vol. 44, no. 2, pp. 357-363, 1999.

[9] Y.-G. Leu, T.-T. Lee, and W.-Y. Wang, "Observer-based adaptive fuzzy-neural control for unknown nonlinear dynamical systems," IEEE Transactions on Systems, Man, and Cybernetics, vol. 29, no. 5, pp. 583-591, 1999.

[10] J. T. Wen and D. S. Bayard, "New class of control laws for robotic manipulators - part 1: non-adaptive case," International Journal of Control, vol. 47, no. 5, pp. 1361-1385, 1988. 